Supporting Information for

\title{
Generation of Aligned Electrospun Fibers by Using Insulating and
}

\section{Hydrophobic Collectors}

Kui Wang, Haiying Tan, Di Tian, Bijin Xiong, Lianbin Zhang, * and Jintao Zhu*

Key Laboratory of Material Chemistry for Energy Conversion and Storage, Ministry of Education (HUST), School of Chemistry and Chemical Engineering, Huazhong University of Science and Technology (HUST), Wuhan 430074, China

*Corresponding authors. Email: jtzhu@mail.hust.edu.cn (J. Z.); zhanglianbin@hust.edu.cn (L.

Z.) 


\section{Supporting figures:}

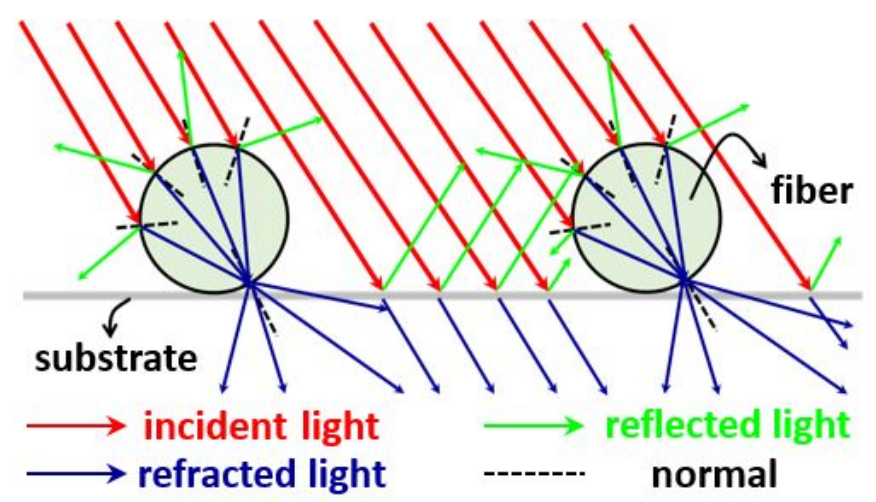

Figure S1. Schematic illustration showing the divergence of light by aligned fibers along with a direction that perpendicular to the long axes of fibers. 


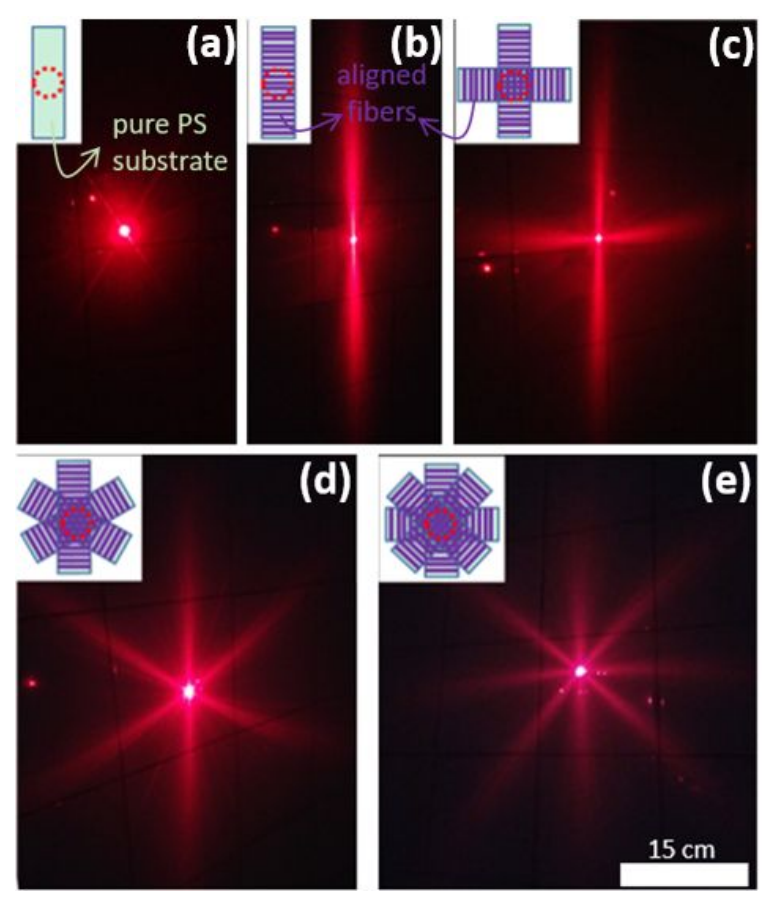

Figure S2. Photographs of the patterns on the plane behind the collector when the laser perpendicularly passed through pure PS collector (a), PS strip covered with aligned fibers (b) and multiple PS strips covered with aligned fibers (c-e). Insets illustrate patterned PS strips with aligned fibers. The light green substrates represent pure PS substrates, the aligned purple lines on the substrates represent aligned fibers, and the scale bar in the last image can be applied to the others. 


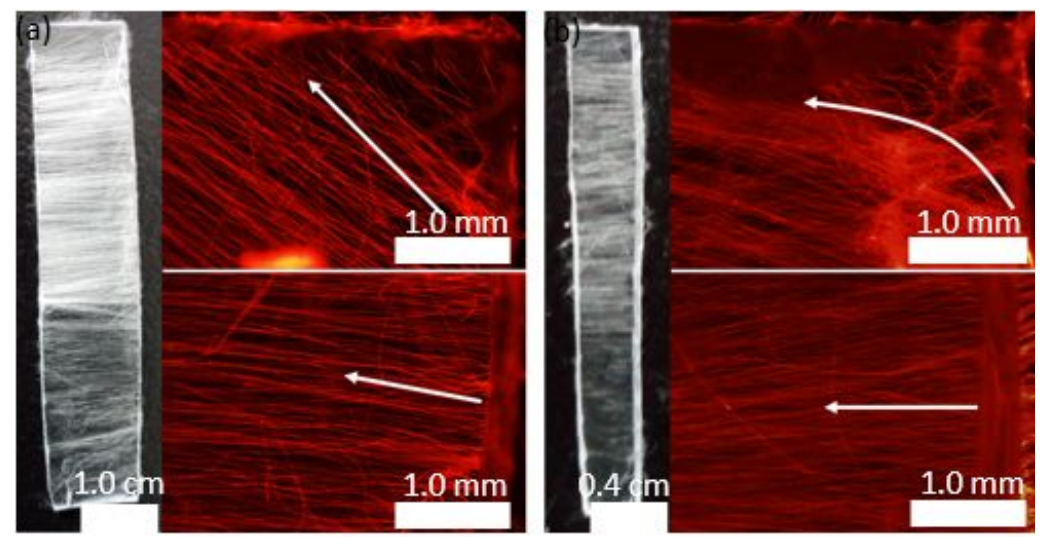

Figure S3. Photographs and fluorescent microscopy images of fibers deposited on the rectangular PS slices with widths of $\sim 1.1 \mathrm{~cm}$ (a) and $\sim 0.3 \mathrm{~cm}$ (b). The arrows in the fluorescent microscopy images indicate the orientation of fibers at the vertices and near the long sides of the rectangular slices. 


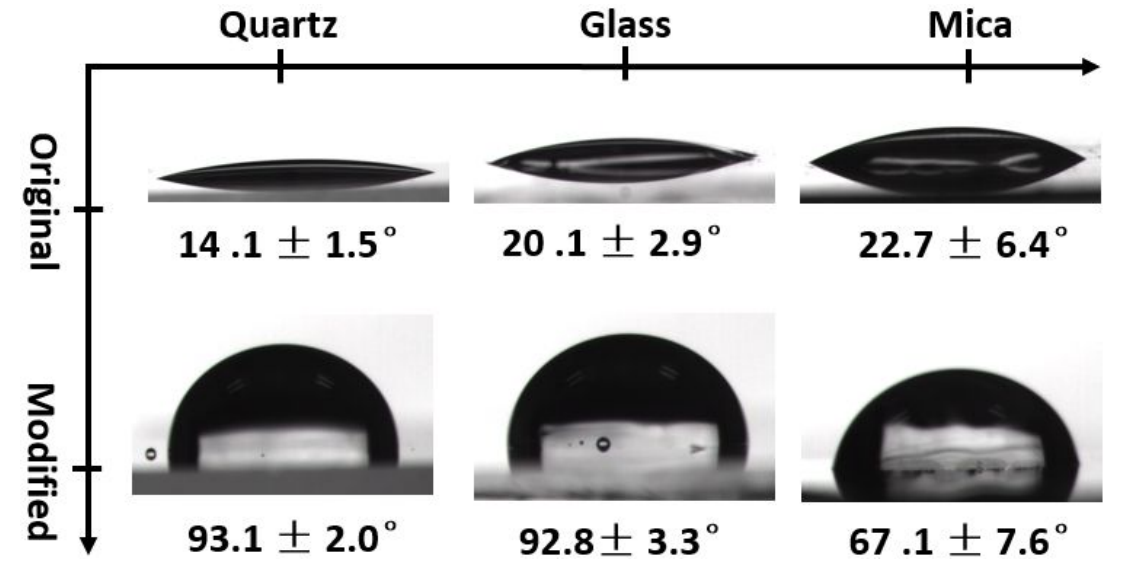

Figure S4. Representative optical microscopy images of static water contact angles of the original and the triethoxy(octyl)silane treated surfaces. 


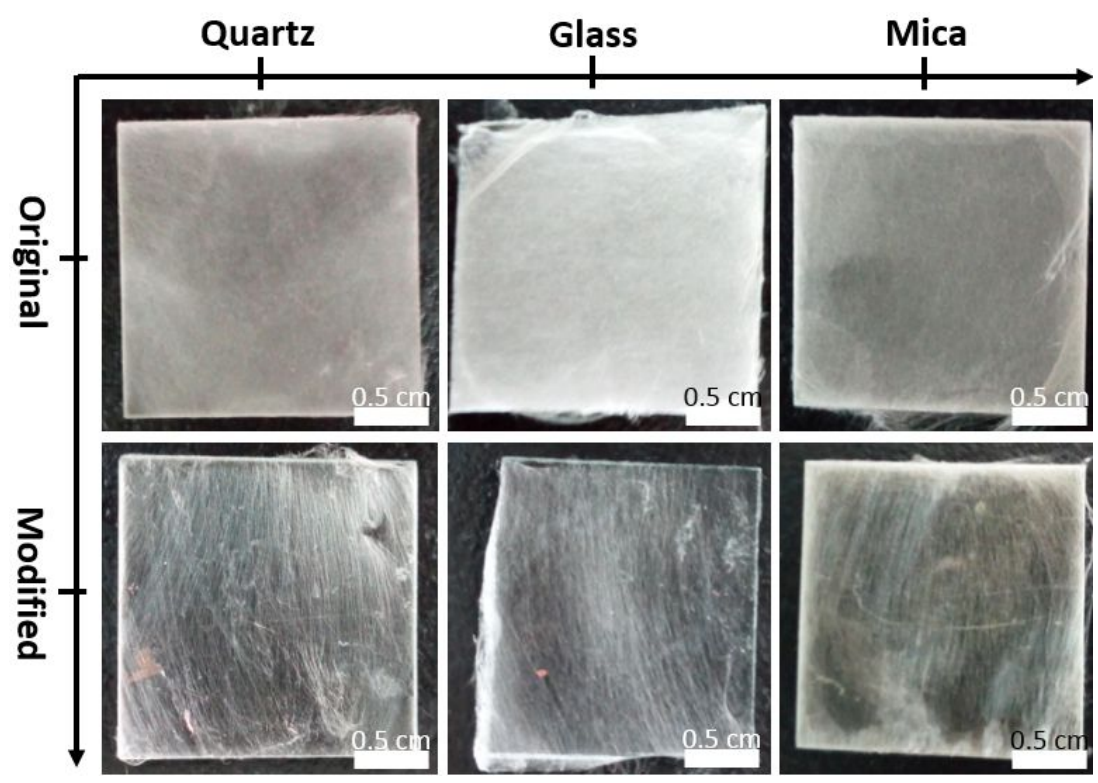

Figure S5. Photographs of the electrospun fibers collected on the original and treated quartz, glass, and mica surfaces. 

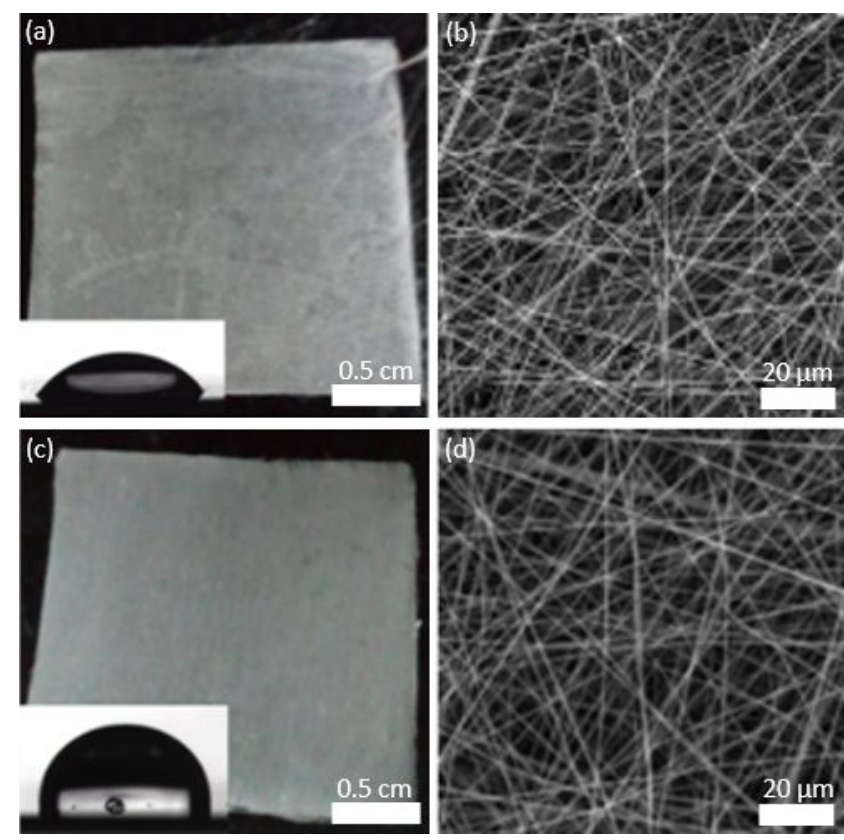

Figure S6. Photographs and SEM images of the electrospun fibers collect by using untreated (a, b) and triethoxy(octyl)silane-modified (c, d) silicon wafer as collectors. Insets in (a, c) display the representative images of static water contact angles. 